\title{
DECAY TO EQUILIBRIUM FOR JUMP PROCESSES WITH HEAVY TAILS
}

\author{
JOSÉ LUÍS DA SILVA \\ Centro de Ciéncias Matemáticas, University of Madeira, Campus da Penteada \\ 9020-105 Funchal, Madeira, Portugal. \\ luis@uma.pt \\ TOBIAS KUNA \\ Department of Mathematics, University of Reading, \\ PO Box 220, Reading, Berkshire, \\ RG6 $6 A X, U K$ \\ t.kuna@reading.ac.uk
}

\begin{abstract}
The case is made for a more careful analysis of the large time asymptotic of infinite particle systems in the thermodynamic limit beyond zero density. The insufficiency of current analysis even in the model case of free particles is demonstrated. Recent advances based on more sophisticated analytical tools like functions of mean variation and Hardy spaces are sketched.
\end{abstract}

Keywords: Large time asymptotic, hydrodynamic limit, infinite particle systems, bounded mean oscillation.

PACS numbers: 11.25.Hf, $123.1 \mathrm{~K}$

\section{Introduction}

Infinite particle systems describe a multitude of phenomena including a description of matter from first principles, but are not limited to these. In particular, applications in life sciences, meteorology and sociology require a good understanding of the dynamic of infinite particle systems far from equilibrium. Few mathematical rigorous results have been obtained for the microscopic dynamic in the thermodynamic limit on microscopic scale even on the mere level of existence results, see Ref. 1 . The complexity of the full system makes it necessary to describe the system by few relevant characteristics like the expected local density and its correlations. Such an approach speeds up the calculation but, more important, it yields a better qualitative and approximative understanding.

In this paper, we aim at explaining which kind of analytical challenges one faces if one wants to describe such systems on a microscopic scale for a large (infinite) system. We argue that even in the case of free dynamics there are unresolved issues 
and a careful reevaluation and a development of analytic techniques is necessary. For interacting systems the equation of motion for the local density would couple with the correlations of the local density. The equation of motion for the correlation would couple with higher correlations and so on. One arrives at an infinite hierarchy of equations. Little is known about the solutions of the full hierarchy, cf. Ref 1 for an overview and for some recent result see Ref. 2, 3, 4, 5 and references therein. In practice, this problem is circumvented by cutting the hierarchy, that is by replacing the correlation by a functional of the local density. One may consider the free systems as the only special case of such cutted hierarchies which are at the same time a rigorous description of a full infinite particle system. The free systems are thus an ideal testbed for a thorough development of the analytic framework necessary for the rigorous treatment of cutted hierarchies.

We do not expect any interesting phenomena in the free case which are not intuitively clear outright from the beginning. What is less clear is how to derive these intuitions from first principles. The main aim of the paper is to convince the reader that this is not merely a technical problem, but that the interesting phenomena are hidden in singularities of the Fourier transform. These singularities are of an unusual type and little attention has been given to them so far. The content of the paper is one approach of treating these singularities in details. We line out new techniques and structures which this treatment requires and develop a conceptually simple and direct proof for the large time asymptotic. In this paper, we consider the special case of jump processes which gives rise to non-trivial heavy tail behavior. The results are stated in a way that they should be generalizable to more general dynamics.

In Section 2, we give a non-rigorous derivation of the description of the dynamics in terms of the local density and we derive different equivalent descriptions of the dynamics. In Section 3, we introduce the particular jump type dynamics, which we consider in this paper, and we derive a solution to the time development of the local density via Fourier transform. In Section 4, we apply Fourier transform techniques and show that the naive use of these techniques only describes systems with zero density adequately. We show that each constant density is an invariant state. A more sophisticated consideration allows us to show that the arithmetic mean of the density indicates the basin of attraction for a given density profile. Unfortunately, more sophisticated techniques are needed to obtain a description which could be generalizable to interacting systems. In Subsection 5, we show that asymptotically (for large time) all our jump processes are comparable to $\alpha$-stable processes. We identify the functions of bounded mean oscillation as the right framework and give an outline of the proof of the general result. In Section 6 , we discuss the implications of this result for the asymptotic of the full distribution of infinite particle systems. We discuss that even for general initial distributions with non-trivial correlation structure (including initial distributions in the presence of a phase transition) the infinite particle system relaxes to equilibrium, that is, all correlations cease. 


\section{Heuristic derivation of the evolution equation}

We consider a system of identical particles in $\mathbb{R}^{d}$. In this paper, we take the standpoint to describe the system by its local density $\rho: \mathbb{R}^{d} \longrightarrow[0, \infty)$, that is $\rho(x)$ describes the density of particles at the point $x$. We need to assume as minimal regularity that $\rho$ is locally bounded, i.e. around each point there exists a ball on which $\rho$ is bounded.

The time development of the density for a free system has to fulfill the equation

$$
\frac{d \rho_{t}}{d t}=A^{*} \rho_{t}
$$

where $A^{*}$ is the operator describing the movement of a single particle. We will heuristically derive this. The reader familiar with this rather straight forward derivation should skip this section. Let us assume that a single particle follows a trajectory $t \mapsto x_{t}$ which is the solution of

$$
\frac{d x_{t}}{d t}=v\left(x_{t}\right)
$$

where $v$ is a vector field on $\mathbb{R}^{d}$ (bounded and Lipschitz). The flow associated to equation (2) is the mapping $\Phi_{t}$ which maps each $x$ to $x_{t}$, where $x_{t}$ is the solution of (2) for the initial condition $x_{0}=x$.

For any bounded subset $\Lambda \subset \mathbb{R}^{d}$, the expression $\int_{\Lambda} \rho_{t}(x) d x$ denotes the number of particles in $\Lambda$ at time $t$. As all particles which are at time $t$ in $\Lambda$ have to have moved from $\Phi_{-t}(\Lambda)$, by conservation of mass, we have

$$
\int_{\Lambda} \rho_{t}(x) d x=\int_{\Phi_{-t}(\Lambda)} \rho(x) d x .
$$

By the transformation formula $\rho_{t}(x)=\rho\left(\Phi_{-t}(x)\right) \operatorname{det}\left(D \Phi_{-t}(x)\right)^{-1}$, where $D \Phi$ denotes the matrix of the derivatives with respect to $x$. One can extend (3) to general functions $\varphi$ on $\mathbb{R}^{d}$

$$
\int_{\mathbb{R}^{d}} \varphi(x) \rho_{t}(x) d x=\int_{\mathbb{R}^{d}} \varphi\left(\phi_{t}(x)\right) \rho(x) d x .
$$

To require (4) for a collection of function $\varphi$, which contains enough functions to approximate an arbitrary function, identifies $\rho_{t}$ uniquely. The characterization via (4) has the advantage that one does not need the time-reverse flow, hence (4) can be directly extended to random movements and singular flows.

For a function $\varphi$, let us define, the new function $\varphi_{t}(x):=\varphi\left(\Phi_{t}(x)\right)$. If $\varphi_{t}$ is $t$-differentiable, then one can easily derive that

$$
\frac{d}{d t} \varphi_{t}(x)=v(x) \nabla \varphi_{t}(x) .
$$

The equation has form (1) for $A \varphi:=v(x) \nabla \varphi$, namely

$$
\frac{d}{d t} \varphi_{t}=A \varphi_{t}, \quad \varphi_{0}:=\varphi .
$$


$A$ is the Liouville operator of the system and (6) is called the Kolmogorov equation. Finally, we can thus write an equation for the time development of the density in weak form: for all smooth $\varphi$ with bounded support holds

$$
\frac{d}{d t} \int_{\mathbb{R}^{d}} \varphi(x) \rho_{t}(x) d x=\int_{\mathbb{R}^{d}} A \varphi(x) \rho_{t}(x) d t .
$$

For such $\varphi$ all operations in (7) are rigorous. Formally, this is equivalent (via integration by parts if $\rho_{t}$ is regular enough) to the master equation $\frac{d}{d t} \rho_{t}=\nabla\left(v \rho_{t}\right)=: A^{*} \rho_{t}$.

\section{Jump type dynamics}

In this paper, we concentrate on systems of particles which move by random jumps. More precisely, each particle jumps independently and the time intervals between jumps are also independent and exponentially distributed with rate $\lambda$. If a particle jumps, it jumps with probability density $\tilde{a}(y-x)$ from $x$ to $y$. One can easily show that the associated Liouville operator is given by

$$
A \varphi(x)=\lambda \int_{\mathbb{R}^{d}} \tilde{a}(y)(\varphi(x+y)-\varphi(x)) d y .
$$

For convenience, we work with $a:=\lambda \tilde{a}$. Analogously to the calculation in Section 2, one can then define the time-development of the local density as in (7). Using Fourier transform one can solve for any smooth $\varphi$ with bounded support the equation (6)

$$
\varphi_{t}(x)=\frac{1}{(2 \pi)^{d / 2}} \int_{\mathbb{R}^{d}} e^{i k x} e^{t(2 \pi)^{d / 2}(\hat{a}(k)-\hat{a}(0))} \hat{\varphi}(k) d k,
$$

where $\hat{a}, \hat{\varphi}$ respectively, denote the Fourier transform of $a$, of $\varphi$ respectively. Furthermore, $\hat{a}$ is a continuous complex valued function which decays to zero at infinity. In addition, one sees easily that the real part of $\hat{a}(k)-\hat{a}(0)$ is strictly negative for $k \neq 0$ and zero at $k=0$. By duality one obtains immediately also the solution for the time development of the density

$$
\rho_{t}(x)=\frac{1}{(2 \pi)^{d / 2}} \int_{\mathbb{R}^{d}} e^{i k x} e^{t(2 \pi)^{d / 2}(\hat{a}(-k)-\hat{a}(0))} \hat{\rho}(k) d k .
$$

If one wants to formulate the time development without reference to the Fourier transform, then one has to introduce the (transition) probabilities $\mu_{t}$ as

$$
\hat{\mu}_{t}(k):=e^{t(2 \pi)^{d / 2}(\hat{a}(k)-\hat{a}(0))} .
$$

According to the theory of negative definite function, $\mu_{t}$ is a probability measure. Then one can write $\varphi_{t}(x)=\int_{\mathbb{R}^{d}} \varphi(x+y) d \mu_{t}(y)$ and $\rho_{t}(x):=\int \rho(x-y) d \mu_{t}(y)$. Explicit formulas for $\mu_{t}$ exist only in special cases.

\section{Large time asymptotic}

In order to get a feeling, how powerful the solution described in (9) is, we will try to derive the large time behavior of the density from it. The Fourier transform of 
the transition probability converges to zero for $k \neq 0$ as the real part of $\hat{a}(k)-\hat{a}(0)$ is strictly negative,

$$
\lim _{t \rightarrow \infty} e^{t(2 \pi)^{d / 2}(\hat{a}(k)-\hat{a}(0))}=\left\{\begin{array}{l}
0, \text { for } k \neq 0 \\
1 \text { for } k=0
\end{array}\right.
$$

and is uniformly bounded by 1. Lebesgue's dominated convergence theorem implies

$$
\begin{aligned}
\lim _{t \rightarrow \infty} \varphi_{t}(x) & =\lim _{t \rightarrow \infty} \int_{\mathbb{R}^{d}} e^{i k x} e^{t(2 \pi)^{d / 2}(\hat{a}(k)-\hat{a}(0))} \hat{\varphi}(k) d k \\
& =\int_{\{0\}} e^{i k x} e^{t(2 \pi)^{d / 2}(\hat{a}(k)-\hat{a}(0))} \hat{\varphi}(k) d k=0 .
\end{aligned}
$$

In the same way, one seemingly get that also $\rho_{t}$ converges to zero for any initial condition $\rho$.

A density $\rho$ is called invariant under the dynamic if $\rho_{t}=\rho$ is a solution of (7). Clearly, all constant functions $\rho$ are invariant, as $\int_{\mathbb{R}^{d}} \rho d \mu_{t}(y)=\rho \mu_{t}\left(\mathbb{R}^{d}\right)=\rho$. Therefore, the result (suggested above) that $\rho_{t} \rightarrow 0, t \rightarrow \infty$ cannot be true in general. The gap in the previous consideration is that $\hat{\rho}(k)$ is in general not a function. For example, if $\rho$ is a constant function, then $\hat{\rho}(k)=(2 \pi)^{d / 2} \rho \delta_{0}(k)$, where $\delta_{0}(k)$ denotes the $\delta$ function which is only a generalized function, more precisely a probability measure. In this case

$$
\rho_{t}(x)=\frac{1}{(2 \pi)^{d / 2}} \int_{\mathbb{R}^{d}} e^{-i k x} e^{t(2 \pi)^{d / 2}(\hat{a}(-k)-\hat{a}(0))}(2 \pi)^{d / 2} \rho \delta_{0}(k) d k=\rho .
$$

This shows that the previous consideration (14) only applies to system which have overall density zero.

This raises the task to extend the large time asymptotic to a wider class of densities $\rho$ which contains in particular the constant functions. Each constant function will be a stable point for the dynamics. Hence it would be desirable also to identify the basin of attraction for each stable point.

Using the same technique as above, the largest class of densities $\rho$ we can treat is the class of all $\rho$ such that its Fourier transform is a signed Radon measure, see Ref. 6. In this case it holds that

$$
\lim _{t \rightarrow \infty} \rho_{t}(x)=\frac{1}{(2 \pi)^{d / 2}} \hat{\rho}(\{0\}) .
$$

Let us illustrate this in a few examples:

(i) If $\rho$ is a constant function then the limit is $\rho$.

(ii) If $\rho$ decays to zero, i.e., for every $\varepsilon>0$ there exists an $R>0$ such that $|\rho(x)| \leq \varepsilon$ for $x$ with $|x|>R$, then the limit is zero.

(iii) If $\rho \in L^{p}\left(\mathbb{R}^{d}, d x\right), p \in[1, \infty)$, then the limit is zero.

(iv) If $\rho(x)=1+\varepsilon \sin \left(x_{1}\right)$, then the limit is zero.

However this does not describe the situation completely, as it is shown by the following example. 
(v) Given $\rho_{0}, \rho_{1} \geq 0$, let $\rho$ be the function defined at each $x=\left(x_{1}, \ldots, x_{d}\right) \in \mathbb{R}^{d}$ by

$$
\rho(x)=\left\{\begin{array}{l}
\rho_{1} \text { if } x_{1} \geq 0 \\
\rho_{0} \text { otherwise }
\end{array} .\right.
$$

In the one dimensional case the Fourier transform is the following generalized function $\hat{\rho}(k)=\sqrt{2 \pi}\left(\rho_{0}+\rho_{1}\right) / 2 \delta_{0}(k)+i\left(\rho_{0}-\rho_{1}\right) / \sqrt{2 \pi} \mathcal{P}(1 / k)$, where $\mathcal{P}(1 / k)$ denotes the Cauchy principal value of $1 / k$. Note that in this case $\hat{\rho}$ is not a signed measure.

So all $\rho$ which have the same value for $\hat{\rho}(\{0\})$ will converge to the same limit. Note that $\hat{\rho}(\{0\})$ is not the same as the value of the Fourier transform at zero. Can one express $\hat{\rho}(\{0\})$ directly in terms of $\rho$ without using Fourier transform. Indeed, one can show that

$$
\frac{1}{(2 \pi)^{d / 2}} \hat{\rho}(\{0\})=\lim _{R \rightarrow+\infty} \frac{1}{\operatorname{vol}(B(R))} \int_{B(R)} \rho(x) d x,
$$

where $B(R)$ denotes the ball of radius $R$ with center zero. This is just the overall density of the system, which we will denote by mean $(\rho)$. A more careful calculation shows that, in example (v) above we have that indeed $\rho_{t}$ converges to $\lim _{R \rightarrow+\infty} \frac{1}{\operatorname{vol}(B(R))} \int_{B(R)} z(x) d x=\frac{\rho_{0}+\rho_{1}}{2}$.

\subsection{Concrete criteria of convergence}

At a first glance, one may think that the previous consideration gives a full answer to the large time asymptotic. If one considers concrete $\rho$, one sees very quickly that it is actually very difficult to check that $\hat{\rho}$ is a measure. Unfortunately, there is no known criterion (at least to our knowledge) characterizing this condition directly in terms of $\rho$ without referring to the Fourier transform. Even more important, a potential extension to interacting systems seems hopeless for such an non-concrete condition, because in the interacting case the Fourier transform will not be computable any longer. Having this in mind, it is desirable to have a clear analytical framework, that is, to identify the correct metric on local densities which characterizes large time behavior.

A first guess for such a metric, which may come to mind, is the uniform metric on bounded $\rho$. This framework is not completely satisfactory, because boundedness is not necessary for the existence of the mean and not any bounded $\rho$ has a large time asymptotic.

Consider the following slowly oscillating function

$$
\rho(x)=\cos (\ln (1+|x|))+\rho_{0}, \quad x \in \mathbb{R}^{d},
$$

where $\rho_{0}$ is a constant greater or equal to 1 . Then for large $R$ it holds

$$
\frac{1}{\operatorname{vol}(B(R))} \int_{B(R)} \rho(x) d x \sim \frac{d}{\sqrt{1+d^{2}}} \sin (\ln (R+1)+\arctan (d))+\rho_{0} .
$$


In general slowly varying functions will show spurious behavior.

Summarizing, we have to find norms or functionals on local densities such that they characterize

- which local densities have a convergent mean,

- which local densities are in the same basin of attraction, i.e., when two local densities converge to the same limit for large time.

Obviously, one norm or functional will not be able to fulfill both requirement simultaneously. Indeed, we will use a semi-norm.

\section{Comparison theorem}

As we pointed out in the previous section, the arithmetic mean does not exists for all bounded functions, hence there is no clear limit point to which these densities may converge at large times. It is even a question if there exists a time asymptotic at all. For a particular class of processes, the large time behavior is rather easy to understand. Let $\alpha \in(1,2], D>0$ be given and define the following semi-group of transition probability densities $\gamma_{t}^{\alpha, D}$ via their Fourier transform

$$
\widehat{\gamma_{t}^{\alpha, D}}(k)=e^{-D t|k|^{\alpha}} \text {. }
$$

Note that $\gamma_{t}^{\alpha, D}(x)=\frac{1}{(D t)^{1 / \alpha}} \gamma_{1}^{\alpha, 1}\left(x /(D t)^{1 / \alpha}\right)$ and $\gamma_{1}^{\alpha, 1}$ is a continuous integrable function that decays to zero at infinity. The constant $D$ only rescales the time. These transition probabilities are $\alpha$-stable distributions and define an $\alpha$-stable Lévyprocesses. In analogy to (10) holds

$$
\rho_{t}(x)=\int_{\mathbb{R}^{d}} \rho(x-y) d \gamma_{t}^{\alpha, D}(y)=\rho * \gamma_{t}^{\alpha, D}(x),
$$

where $*$ denotes the convolution. The right hand side is already a generalized mean defined as follows: Let $f$ be a normalized non-negative continuous function on $\mathbb{R}^{d}$. Then the arithmetic mean associated to $f$ is defined as

$$
\operatorname{mean}_{f}(\rho):=\lim _{R \rightarrow \infty} \int_{\mathbb{R}} \rho(x) \frac{f(x / R)}{R^{d}} d x,
$$

if this limit exists. Note that the growth of $R$ depends on $t$, namely $(D t)^{1 / \alpha}$. We see that the large time limit of $(19)$ is just mean $_{\gamma_{1}^{\alpha, 1}}(\rho)$ if and only if the limit exists. In particular, one can show that the limit in (19) will be independent of $x$. It remains open to what extend $\operatorname{mean}_{f}(\rho)$ depends on the choice of $f$. Similar considerations as in (4) show that for integrable $\rho$ all these means are zero independent of $f$. However, one cannot expect that this is true for all densities in general.

To treat general jump dynamics as introduced in (8), we derived, motivated by a paper of R. L. Dobrushin and Ra. Siegmund-Schultze ${ }^{7}$, a comparison result with $\alpha$-stable processes see Ref. 6 and 8. R. L. Dobrushin and Ra. Siegmund-Schultze derived the large time asymptotic of the full infinite particle system, cf. Section 6 , under the assumption that a comparison results holds for $\alpha=2$. For jump processes 
this comparison result was derived in Ref. 6 for the case $\alpha=2$ and furthermore, a more elementary and general proof of the convergence result for the infinite particle process was given. Comparison results for general $\alpha$ will be given which are sufficient to yield convergence of the infinite particle process, see Ref. 8 .

First we have to identify to which $\alpha$-stable process we want to compare the jump process. Let $a$ be such that there exists an $\alpha \in(1,2]$ such that

$$
\lim _{k \rightarrow 0} \frac{\Re(\hat{a}(k))-\hat{a}(0)}{|k|^{\alpha}}=-D .
$$

The main comparison estimate is the following

Lemma 5.1. For any integrable $\varphi$ holds (uniform in the $L^{1}$-norm of $\varphi$ ) that

$$
\lim _{t \rightarrow \infty} \frac{1}{t^{d / \alpha}} \sup _{x \in \mathbb{R}^{d}}\left|\varphi_{t}(x)-\gamma_{t}^{\alpha, D} * \varphi(x)\right|=0 .
$$

The proof is based on a careful estimation of the Fourier transform using that $\varphi$ is sufficiently regular. Modulo an error exponential in time, only frequencies of order less or equal to 1 are important.

This result gives us a control of the large time asymptotic for $x$ of order $t^{1 / \alpha}$. More precisely, the above estimate implies that

$\lim _{c \rightarrow 0, C \rightarrow \infty} \lim _{t \rightarrow \infty}\left|\int_{c t^{1 / \alpha} \leq|x| \leq C t^{1 / \alpha}} \varphi_{t}(x) \rho(x) d x-\int_{c t^{1 / \alpha} \leq|x| \leq C t^{1 / \alpha}} \gamma_{t}^{\alpha, D} * \varphi(x) \rho(x) d x\right|=0$.

At this point we have to introduce a natural class of $\rho$ for which we want to derive the comparison. All arguments obviously work for bounded $\rho$. However, this class seems not to be natural. For the existence of the arithmetic mean, only local integrability is necessary. The class of function adapted to this type of problems is the class of functions of bounded mean oscillation.

Definition 5.1. A locally integrable function $f$ defined on $\mathbb{R}^{d}$ is said to belong to $\mathrm{BMO}:=\mathrm{BMO}\left(\mathbb{R}^{d}\right)$ (bounded mean oscillation) if the inequality

$$
\frac{1}{\operatorname{vol}(B)} \int_{B}\left|f(x)-f_{B}\right| d x \leq C
$$

holds for all balls $B$ in $\mathbb{R}^{d}$, here $f_{B}:=\operatorname{vol}(B)^{-1} \int_{B} f(x) d x$ denotes the mean value of $f$ over the ball $B$. The smallest bound $C$ for which (24) holds is then taken to be the semi-norm of $f$ in BMO.

Clearly, all bounded functions are in BMO, though not all BMO functions are bounded, they may grow logarithmically. Clearly, bounded mean oscillation seems to be necessary for the existence of the arithmetic means though clearly not sufficient.

Theorem 5.1. For all $\rho \in \mathrm{BMO}$ and all infinite differentiable function $\varphi$ with compact support, holds that

$$
\lim _{t \rightarrow \infty}\left|\int \varphi_{t}(x) \rho(x) d x-\int \gamma_{t}^{\alpha, D} * \varphi(x) \rho(x) d x\right|=0 .
$$


The proof exploits the duality between BMO and the Hardy space in the sense of E. Stein, see Ref. 9. It is very helpful that the maximal operator used in the definition of the Hardy space fits structurally well with the jump type dynamics. Furthermore, one has to show that

$$
\lim _{c \rightarrow 0, C \rightarrow \infty} \lim _{t \rightarrow \infty}\left|\int_{x \leq c t^{1 / \alpha}|x|} \varphi_{t}(x) \rho(x) d x\right|+\left|\int_{|x| \geq C t^{1 / \alpha}} \varphi_{t}(x) \rho(x) d x\right|=0 .
$$

This can be derived by a careful analysis based on the invariance of the constant functions. Unfortunately, this bound is not uniform in the $L^{1}$-norm of $\varphi$. The latter would be sufficient to show the comparison Theorem 5.1 even in the strong sense, that is in the BMO-norm.

It remains to identify the subset of BMO functions for which the arithmetic mean exist. A variation of the definition of functions of vanishing mean oscillation seems to be a promising candidate. Research about BMO and VMO functions typically focus on regularity issues, that is, in Ref. 9 the author is mainly interested in the limit $R \rightarrow 0$. Furthermore, only smooth quickly decaying kernels are used in the definition of the maximal operators. In our application, neither of the two holds. Therefore in order to answer the remaining questions, we have to extend essentially the theory of BMO functions and the associated maximal operators.

\section{Infinite particle system}

Till now we only have considered the description of our particle system solely in terms of one characteristic of the system, that is the density. A natural question to ask is what happens with other characteristics of the system, for example higher correlations. The most general quantity, one may look at, is the probability distribution of the full system of infinite many particles. More precisely, we consider a probability (measure) on the set of all possible configurations of infinite many indistinguishable particles. A single configuration is hence given by a sequence of positions $x_{1}, x_{2}, \ldots$. Two sequences which differ only by a relabeling are considered as identical. Furthermore, we require that in any bounded volume there are at most finite many points. The state space of our system is the collection of all these sequences and the distributions are probabilities on this infinite dimensional space.

As all particles move independently, the overall dynamics is a kind of infinite product of the original dynamic. However, it is non-trivial to show that in any given bounded volume there are not more than finite many particles at any given time. Furthermore, one has to show that in each finite time interval only a finite number of particles pass through each bounded volume. This can only hold under additional conditions on the initial configuration, for details, see Ref. 10.

The invariant distributions for this dynamics are the free gas law for different constant densities. The associated probability is the so-called Poissonian random field. One can show the following extension of the large time asymptotic for the full distribution of the process under very mild conditions. In particular, the result is 
applicable to pure states even in regimes of potential phase transitions.

Proposition 6.1. Let $\mu$ be a probability on all configurations for which the dynamic exists. Assume that $\mu$ has first and second local moments. Assume that $\mu$ fulfills a weak mixing condition (the covariance $u_{\mu}^{(2)}$ decays like $|x|^{-d+\varepsilon}$ ). Furthermore, we assume that the expected local density $\rho_{\mu}$ associated to $\mu$ is BMO. Then, the one-dimensional distribution converges weakly to the Poissonian random field for (constant) density mean $\left(\rho_{\mu}\right)$ when $t$ tends to infinity.

\section{Acknowledgments}

We would like to express our gratitude for the splendid hospitality of our colleagues and friends Victoria Bernido and Christopher Bernido during a very pleasant stay at Jagna during the 6th Jagna International Workshop. Financial support of the project PEst-OE/MAT/UI0219/2011 is gratefully acknowledged. T.K. would like to thank the EPSRC project EP/H022767/1 for its support.

\section{References}

1. Y. G. Sinai (ed.), Ergodic Theory with Applications to Dynamical Systems and Statistical Mechanics, Encyclopaedia Math. Sci. Vol. II (World Scientific, Berlin Heidelberg, 1989), Berlin Heidelberg, ch. Dynamical systems of statistical Mechanics and Kinetic Equations.

2. Y. Kondratiev, O. Kutoviy and E. Zhizhina, J. Math. Phys. 47, 501 (2006).

3. Y. Kondratiev, O. Kutoviy and R. Minlos, J. Funct. Anal. 255, 200 (2008).

4. C. Berns, Y. Kondratiev, Y. Kozitsky and O. Kutoviy, Kawasaki dynamics in continuum: micro- and mesoscopic descriptions, tech. rep., SFB 701, Bielefeld (2011), arXiv:11109.4754.

5. D. Finkelshtein, Y. Kondratiev and O. Kutoviy, Correlation functions evolution for the Glauber dynamics in continuum, tech. rep., SFB 701, Bielefeld (2011), arXiv:1107.3487.

6. Y. G. Kondratiev, T. Kuna, M. J. Oliveira, J. L. Da Silva and L. Streit, Hydrodynamic limits for free Kawasaki dynamics of continuous particle systems, tech. rep., U. Madeira (2009), arXiv:0912.1312v1.

7. R. L. Dobrushin and R. Siegmund-Schultze, Math. Nachr. 105, 199 (1982).

8. J. da Silva and T. Kuna, Large time asymptotic for free jump type process with long tails for infinite particle systems, In preparation, (2012).

9. E. M. Stein, Harmonic Analysis (Princeton University Press, 1993).

10. Y. G. Kondratiev, E. Lytvynov and M. Röckner, Condensed Matter Physics 11, $701(2009)$. 